\title{
IGF-1R nuclear import and recruitment to chromatin involves both alpha and beta subunits
}

\author{
Jack V. Mills ${ }^{1} \cdot$ Eliot Osher ${ }^{1} \cdot$ Guillaume Rieunier $^{1} \cdot \operatorname{lan}$ G. Mills ${ }^{2} \cdot$ Valentine M. Macaulay $^{2,3}$
}

Received: 23 February 2021 / Accepted: 6 April 2021

(c) The Author(s) 2021 OPEN

\begin{abstract}
Mature type 1 insulin-like growth factor receptors (IGF-1Rs) are heterotetrameric structures comprising two extracellular a-subunits disulphide-bonded to two transmembrane $\beta$-subunits with tyrosine kinase activity. IGF-1R is a well-known cell surface mediator of malignant growth, with an incompletely understood role upon nuclear import as a transcriptional regulator. Previous characterisation of nuclear IGF-1R focused on IGF-1Rß. Here, we aimed to clarify the source of nuclear IGF-1R and investigate whether a-subunits contribute to nuclear IGF-1R function. Using prostate cancer cell lines DU145 and 22Rv1 we detected nuclear $\alpha$ - and $\beta$-subunits, with increase in nuclear signal upon IGF-treatment and reduction in response to IGF-1R inhibitor BMS-754807. Following biotinylation of cell surface proteins, biotinylated $a-$ and $\beta$-subunits were detected in nuclear extracts of both cell lines. Furthermore, $a$ - and $\beta$-subunits reciprocally coprecipitated from nuclear extract. Finally, we detected recruitment of both subunits to regulatory regions of chromatin, including the promoter of the oncogene JUN, that we previously identified in ChIP-seq as sites of IGF-1R $\beta$ enrichment. These data confirm the cell surface origin of nuclear IGF-1R, suggest the presence of nuclear $\alpha \beta$ complexes and reveal that both IGF-1 Ra- and $\beta$-subunits contribute to pro-tumorigenic functions of nuclear IGF-1R.
\end{abstract}

\section{Introduction}

Type 1 insulin-like growth factor receptor (IGF-1R) is a ubiquitously expressed, transmembrane receptor tyrosine kinase (RTK), closely related to the insulin receptor (INSR) and the main mediator of IGF related signalling and activity [1-3]. Like INSR, IGF-1Rs are translated as $\sim 220$ kDa pro-receptors containing signal peptide and $\alpha$ - and $\beta$-subunit sequence that are co-translationally inserted into the endoplasmic reticulum (ER). After disulphide-bonding and folding, IGF-1Rs are transported to the trans-Golgi network where proreceptor cleavage generates a-subunits $(\sim 140 \mathrm{kDa})$ and $\beta$-subunits $(\sim 100 \mathrm{kDa})[3]$, forming heterotetrameric holoreceptors in a disulphide-bonded $\mathrm{a}_{2} \beta_{2}$-configuration. These mature receptors undergo trafficking to the plasma membrane, where the extracellular a-subunits form a complex binding site for a single IGF molecule and the $\beta$-subunits, which contain small extracellular and transmembrane domains, transmit signals via the intracellular tyrosine kinase domain [4] (Fig. 1a). Under normal physiological conditions IGF axis signalling is tightly regulated, however the IGF axis has long been recognised for its contribution to cancer growth and metastasis by promoting cell survival, proliferation and invasion [5, 6]. Because of these functions the IGF axis is a target for therapeutic intervention [7]. Despite some cases showing exceptional responses, the majority of trials involving IGF-1R inhibitors have failed, due to factors including lack of

Supplementary Information The online version contains supplementary material available at https://doi.org/10.1007/s12672-02100407-8.

$\triangle$ Valentine M. Macaulay, valentine.macaulay@nds.ox.ac.uk| 'Department of Oncology, University of Oxford, Old Road Campus Research Building, Roosevelt Drive, Oxford OX3 7DQ, UK. ${ }^{2}$ Nuffield Department of Surgical Sciences, University of Oxford, Oxford, UK. ${ }^{3}$ Oxford Cancer and Haematology Centre, Oxford University Hospitals NHS Foundation Trust, Churchill Hospital, Oxford OX3 7LJ, UK.

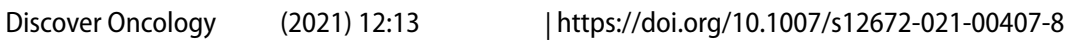


predictive biomarkers, dose-limiting hyperglycaemia resulting from co-inhibition of INSR, and incomplete understanding of IGF-1R biology [8-10].

Work from our group and others revealed that upon activation, IGF-1R, like many other RTKs, can translocate to the nucleus, the initial trafficking step involving clathrin mediated-endocytosis [11-14]. We also reported that nuclear IGF-1R undergoes ligand-induced recruitment to regulatory regions of chromatin, and interacts with RNA Polymerase II (RNAPollI) to promote RNAPolll recruitment and expression of genes including JUN that drive tumour cell proliferation and migration [15]. Others find that nuclear IGF-1R also interacts with transcription factors and nuclear proteins to alter their function [16-18].

Data from Larsson and colleagues indicate that IGF-1R translocation requires SUMOylation of 3 conserved $\beta$-subunit lysine residues followed by an importin- $\beta$ /RANBP2-meditated nuclear import mechanism [12, 14]. We found that nuclear IGF-1R is more frequently detected in malignant than benign epithelium, and is associated with advanced tumour stage in prostate cancer and reduced overall survival in renal cancer $[11,15]$. There is also evidence that nuclear IGF-1R associates with response to IGF1R antibody therapy in patients with sarcoma [19], suggesting that nuclear IGF-1R may indicate dependence on the IGF axis.

The majority of this work to elucidate nuclear IGF-1R function has focussed on detection of the $\beta$-subunit only, so it is unclear whether the receptor $\alpha$-subunit also undergoes translocation and whether it is involved in the nuclear function of IGF-1R. While our lab and others were previously able to detect full-length IGF-1 Ra- and $\beta$-subunits in the nucleus by subcelIular fractionation and immunohistochemistry $[11,12,20]$, it is unclear to what extent the $\alpha$-subunit contributes to the nuclear function of IGF-1R. Here, the objective was to determine whether there is a functional role of the IGF-1Ra-subunit in the nucleus, distinct or otherwise from the $\beta$-subunit, aiming to further elucidate the biology of nuclear IGF-1R and understand its contribution to cancer biology as well as its potential as a therapeutic target.

In this study we used two prostate cancer cell lines, DU145 and 22Rv1, that are respectively androgen receptor (AR) negative and positive, a receptor whose function is critical for prostate cancer progression [21,22] and with which there is known cross-talk with the IGF-axis [23, 24]. We used subcellular fractionation to detect both $\alpha$ - and $\beta$-subunits in the nucleus of both DU145 and 22Rv1 prostate cancer cells and showed by immunofluorescence microscopy that the $\alpha$-subunit of nuclear IGF-1R is responsive to IGF-1R inhibition, analogous to previous results for the $\beta$-subunit [11]. Using cell surface protein biotinylation, we confirmed that both IGF-1R subunits detected in the nucleus had originated from the cell surface, confirming a widely accepted hypothesis in the field. We also showed, via co-immunoprecipitation from nuclear extract, that the two nuclear subunits interact with each other, suggesting that IGF-1R may undergo translocation as holoreceptors or a $\beta$ half receptors. We also detected recruitment of the IGF-1 Ra-subunit to previously identified IGF-1R 3 -subunit chromatin binding sites, suggesting that IGF-1Ra-subunit is also involved in the nuclear function of the receptor.

\section{Methods}

\subsection{Cell lines}

DU145 (from Cancer Research UK Clare Hall Laboratories) and 22Rv1 prostate cancer cells (obtained from Professor Sir Walter Bodmer, University of Oxford) were cultured in RPMI 1640 medium with $10 \%$ fetal calf serum (FCS). Both were mycoplasmafree when tested with MycoAlert (Lonza Rockland Inc.). DU145 and 22Rv1 cells were authenticated by STR genotyping in 2016 (by Eurofins Medigenomix Forensik GmbH and Cancer Research UK Clare Hall Laboratories respectively). After confirming cell line identity, early passage stocks were expanded and cryopreserved, and used within 20 passages of recovery.

\subsection{Western blotting and antibodies}

Cell fractions and whole cell extracts (WCE, prepared in IGF-1R lysis buffer as described in [11]) were resolved by SDSPAGE and western blotting using antibody for IGF-1 Ra [Santa Cruz Biotech. (Heidelberg, Germany), sc-712], IGF-1R [Cell Signalling Technology (Leiden, The Netherlands), CST-3027], EGFR (D38B1) XP (Cell Signalling Technology, CST-4267), AR (Cell Signalling Technology, CST-5153), Lamin A/C (BD Biosciences (Wokingham, UK) 612162) and $\beta$-tubulin (Cell Signalling Technology, CST-86298).

\subsection{Subcellular fractionation}

Cells were fractionated into nuclear (N) and cytoplasmic (C) fractions using NE-PER ${ }^{\mathrm{TM}}$ Nuclear and Cytoplasmic Extraction Reagent kit (ThermoFisher (Paisley, United Kingdom) Cat. No. 78833) as per manufacturers protocol with minor 
modifications. Briefly, following appropriate treatment, cells were collected by cell scraping in PBS, pelleted by centrifugation at $1200 \mathrm{rpm}, 5 \mathrm{~min}$ at $4{ }^{\circ} \mathrm{C}$ and incubated for $15 \mathrm{~min}$ on ice in Cytoplasmic Extraction Reagent (CER) I following $1 \mathrm{~min}$ vigorous vortexing. Following addition of CER II, samples were vortexed vigorously for $20 \mathrm{~s}$ and incubated on ice for a further $3 \mathrm{~min}$. Samples were again vortexed vigorously for $20 \mathrm{~s}$ before centrifugation at $15000 \mathrm{rpm}, 5 \mathrm{~min}$ at $4{ }^{\circ} \mathrm{C}$. Supernatants (cytoplasmic extract) were removed and stored at $-80^{\circ} \mathrm{C}$ until analysis. Pelleted nuclei were washed once in ice cold PBS, before addition of Nuclear Extraction Reagent (NER) and incubation for $1 \mathrm{~h}$ at $4{ }^{\circ} \mathrm{C}$ with agitation. Following incubation samples underwent centrifugation at $15,000 \mathrm{rpm}, 10 \mathrm{~min}$ at $4{ }^{\circ} \mathrm{C}$ and supernatant (nuclear extract) was removed and stored at $-80^{\circ} \mathrm{C}$ until analysis.

\subsection{Cell surface protein biotinylation}

Cells were serum-starved overnight and incubated for 10 min with PBS control or EZ-Link ${ }^{\text {TM }}$ Sulfo-NHS-SS-Biotin label using the Pierce ${ }^{T M}$ Cell Surface Biotinylation and Isolation Kit protocol (ThermoFisher Cat. No. A44390) according to the manufacturer's protocol. Cells were then washed twice with ambient PBS, treated with RPMI-1640 medium supplemented with $10 \% \mathrm{FCS}$ for 20 min at $37{ }^{\circ} \mathrm{C}$ and then fractionated into nuclear $(\mathrm{N})$ and cytoplasmic (C) fractions as described previously. Biotinylated proteins were eluted using NeutrAvadin Agarose spin columns and analysed by western blot alongside N, C and WCE input controls.

\subsection{Co-immunoprecipitation}

Cells were cultured in RPMI-1640 medium with 10\% FCS and fractionated into $\mathrm{N}$ and $\mathrm{C}$ fractions as above. Equal concentrations of nuclear lysates were precleared by incubation with Protein A agarose beads [Merck Millipore (Watford, UK) Cat. No. P9269] and Normal Rabbit IgG antibody (CST-2729) for $1 \mathrm{~h}$ at $4{ }^{\circ} \mathrm{C}$ with rotation. Precleared nuclear extracts were immunoprecipitated overnight at $4^{\circ} \mathrm{C}$ with rotation with antibody for IGF-1 Ra (ThermoFisher (24-60, MA5-13817), IGF$1 \mathrm{R} \beta$ (CST-3027) or Normal Rabbit IgG control (CST-2729). Immunoprecipitated proteins were collected by $2 \mathrm{~h}$ incubation at $4{ }^{\circ} \mathrm{C}$ with rotation with Protein A agarose beads (Merck Millipore (Watford, UK) Cat. No. P9269) and analysed alongside $\mathrm{N}, \mathrm{C}$ and WCE input controls by western blotting.

\subsection{Immunofluorescence staining and microscopy}

Immunofluorescence staining was performed as per Cell Signalling Technology protocol. Briefly, cells were fixed with 4\% paraformaldehyde [methanol free, Alfa Aesar (Heysham, UK) 43368) in ambient PBS for 20 min at room temperature (RT), incubated in Blocking Solution [5\% Bovine Serum Albumin (BSA) and 0.3\% Triton X-100 in ambient PBS] for $1 \mathrm{~h}$ at room temperature. Blocking Solution was removed and cells were incubated overnight at $4{ }^{\circ} \mathrm{C}$ with primary antibodies diluted in Blocking Solution: IGF-1Ra (24-60, MA5-13817, ThermoFisher, 1:250) or IGF-1Rß (CST 9750, 1:500). After washing, cells were incubated in secondary antibody diluted in blocking solution: goat anti-mouse lgG ( $\mathrm{H}+\mathrm{L}$, Highly Cross-Adsorbed Secondary Antibody, Alexa Fluor 488, 1:2000, Invitrogen, ThermoFisher) for cells incubated with primary antibody for the a-subunit, or goat anti-Rabbit lgG (H+L Highly Cross-Adsorbed Secondary Antibody, Alexa Fluor 488 1:2000, Invitrogen, ThermoFisher) for cells incubated with primary antibody for the $\beta$-subunit, for $1 \mathrm{~h}$ at room temperature with protection from light. Cover slips were mounted using mounting medium containing 4',6-diamidino-2-phenylindole (DAPI). Slides were stored at $4{ }^{\circ} \mathrm{C}$ until visualisation on a Zeiss LSM 780 Confocal microscope (Carl Zeiss, Germany) using $\times 63$ magnification objective. Alexa Fluor 488 was excited with laser line $488 \mathrm{~nm}$ and emission collected between 480 and $670 \mathrm{~nm}$. Alexa Fluor 594 was excited with laser line $594 \mathrm{~nm}$ and emission collected between 590 and $745 \mathrm{~nm}$. Nuclear IGF-1R was assessed using FIJ (ImageJ) software by quantifying IGF-1R signal within the boundaries of outlined DAPI stained nuclei.

\subsection{Chromatin immunoprecipitation and qPCR}

Cultured cells were fixed with 1\% formaldehyde before being processed using Merck Millipore ChIP kit (Cat. No. 17-295) as per the manufacturers protocol, with minor modifications as in [15]. Chromatin was pre-cleared using salmon sperm DNA/Protein A agarose slurry (Millipore ChIP kit, Cat. No. 17-295) with Normal Rabbit IgG (CST-2729). Samples were immunoprecipitated using antibody for IGF-1 Ra (ThermoFisher, 24-60, MA5-13817) or IGF-1Rß (CST-3027). Immunoprecipitated proteins were collected using salmon sperm DNA/Protein A agarose slurry (Millipore ChIP kit, Cat. No. 17-295) 

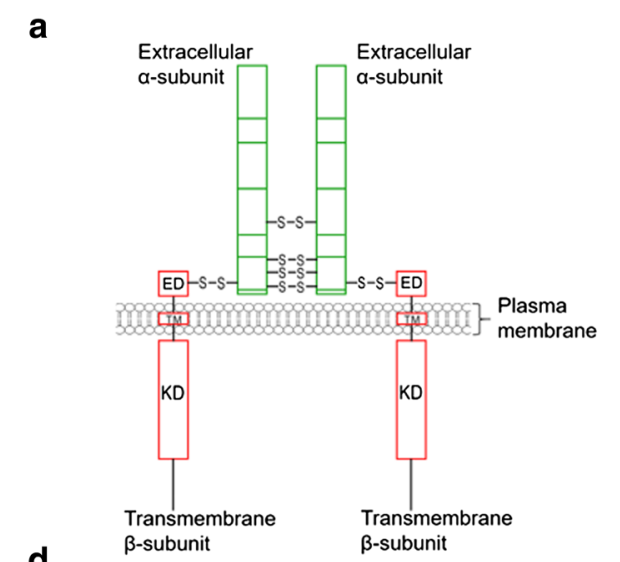$$
\text { d }
$$$$
\begin{array}{ll}
\text { Transmembrane } & \text { Transmembrane } \\
\beta \text {-subunit } & \beta \text {-subunit }
\end{array}
$$
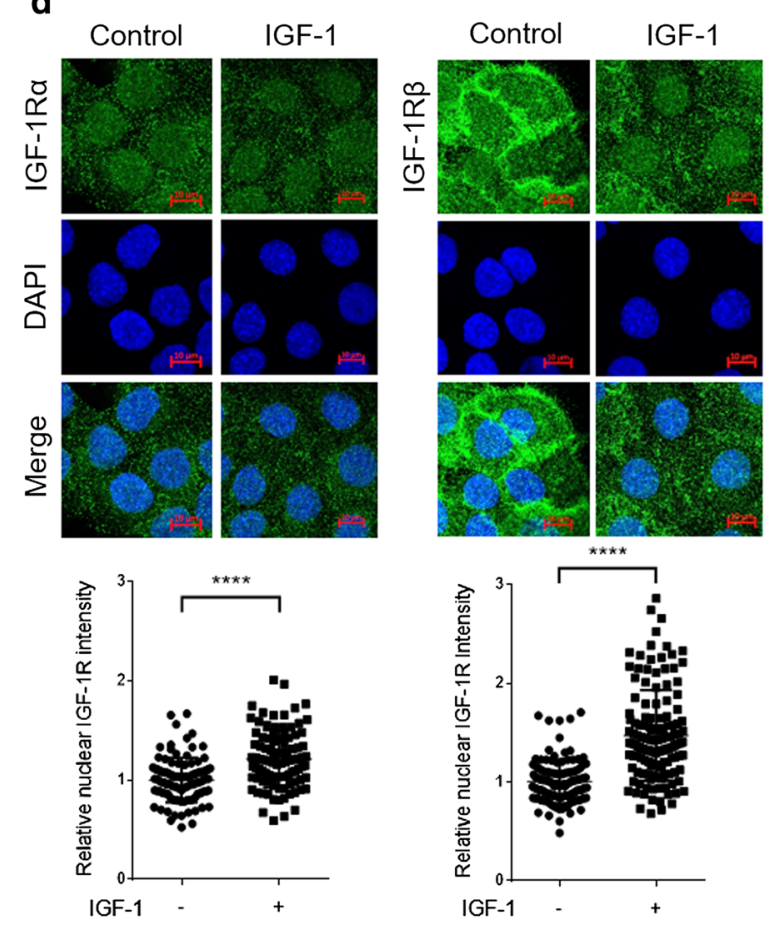

b

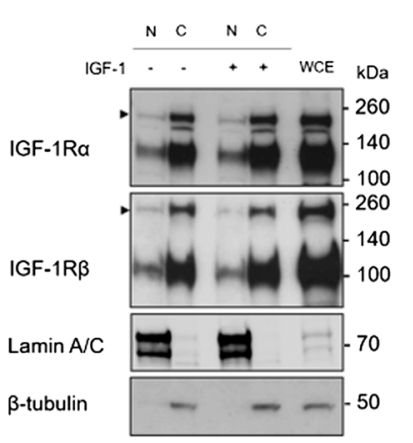

e
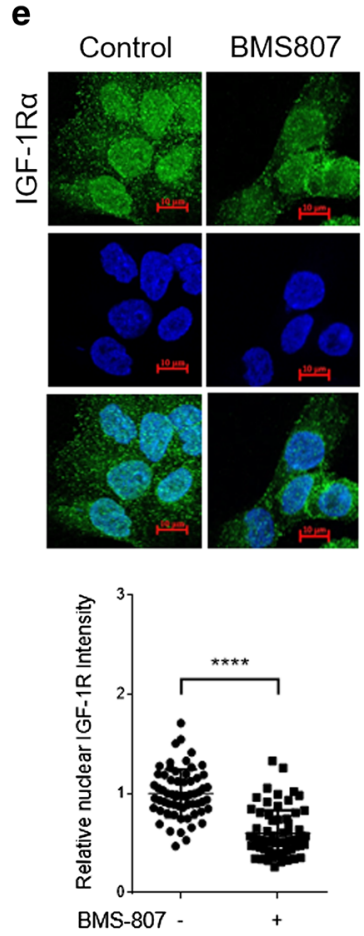

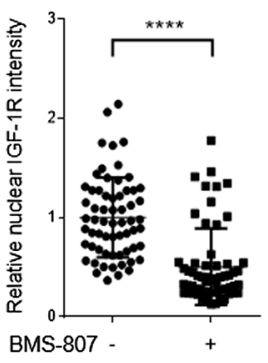

C $22 R v 1$

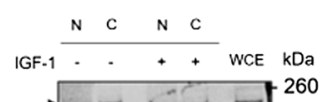

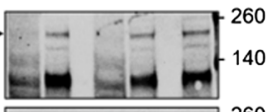

-140
-100

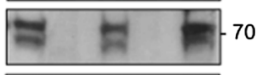

R
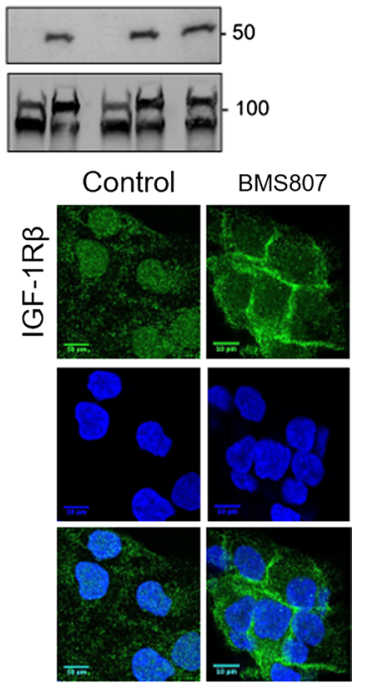

BMS-807 -

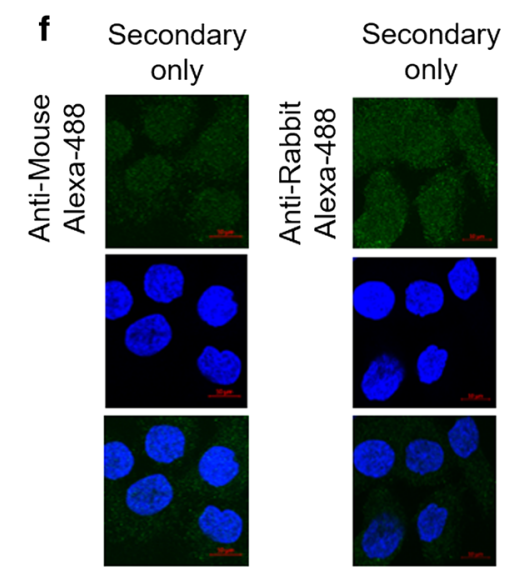


4 Fig. 1 Full length IGF-1Ra- and $\beta$-subunits are detectable in the nucleus of prostate cancer cells a Domain structure of mature IGF-1R at the plasma membrane, showing extracellular a-subunit (green), transmembrane $\beta$-subunit (red), extracellular (ED), transmembrane (TM), kinase domains (KD) and disulphide bonds, modified from [3]. b, c Western blots showing detection of both IGF-1Ra- and $\beta$-subunits in the nucleus of DU145 (b) and 22Rv1 (c) cells. Cells were serum-starved for $24 \mathrm{~h}$, treated with solvent $(4.5 \mu \mathrm{M} \mathrm{HCl})$ or IGF-1 (50 nM) for 20 min before fractionation. All western blots shown are representative of 3 independent biological repeats. Arrowheads: 220 kDa IGF-1R proreceptor. $\mathbf{d}$ Images showing immunofluorescence staining of IGF-1Ra and IGF-1Rß in DU145 cells. DU145 cells were serum-starved and IGF-treated as in b-c followed by immunofluorescent staining. Graphs: mean \pm SD nuclear IGF-1 Ra- and $\beta$-subunits $(n \geq 30$ cells per condition for $n=3$ biological repeats). Data were analysed by unpaired student's t test and quantification of nuclear levels of IGF-1Ra- and $\beta$-subunits showed a significant increase on treatment with IGF-1 $\left.{ }^{*} p<0.05 ;{ }^{* *} p<0.005 ;{ }^{* * *} p<0.001 ;{ }^{* * * *} p<0.0001\right)$ e Images showing IGF-1Ra and IGF-1R $\beta$ immunofluorescence in DU145 cells. Cells were grown in full medium with $10 \%$ FCS and incubated with solvent (0.1\% DMSO) or IGF-1R inhibitor BMS-754807 (100 nM) for $6 \mathrm{~h}$ at $37^{\circ} \mathrm{C}$ and processed for immunofluorescence staining. Graphs: mean \pm SD nuclear IGF-1Ra- and $\beta$-subunits ( $n \geq 30$ cells per condition for $n=2$ biological repeats). Data were analysed by unpaired student's $t$ test and quantification of nuclear levels of IGF-1Ra- and $\beta$-subunits showed a significant decrease on treatment with BMS-754807 $\left({ }^{*} p<0.05 ;{ }^{* *} p<0.005 ;{ }^{* * *} p<0.001 ;{ }^{* * * *} p<0.0001\right)$ f Images showing cultured DU145 cells stained with Alexa-488 secondary antibodies as used in $\mathbf{d}$ and $\mathbf{e}$ in the absence of primary antibody. These images represent a negative control for immunofluorescence staining and demonstrate the specificity of the IGF-1 Ra- and $\beta$-subunit signals shown in $\mathbf{d}$ and $\mathbf{e}$

and processed as per Merck Millipore ChIP kit protocol to generate DNA samples. The qPCR was performed using Luna ${ }^{\circledR}$ Universal qPCR Master Mix (New England Biolabs (Hitchin, UK) Cat. No. M3003) as per the manufacturers protocol using primer sequences for JUN promoter and an intergenic region of chromosome 17 (IntChr17) described in [15].

\section{Results and discussion}

\subsection{Both IGF-1Ra- and $\beta$-subunits are detectable in the nucleus of prostate cancer cells}

DU145 and 22Rv1 prostate cancer cell lines showed detectable levels of nuclear IGF-1Ra- and IGF-1R $\beta$-subunits in nuclear extracts prepared from cells cultured in both serum-starved and IGF-treated conditions (Fig. 1b, c). There was no evidence of significant change in nuclear IGF-1R content upon IGF-1 stimulation, possibly reflecting non-linearity of western blotting. We previously used immunofluorescence to assess IGF-induced changes in IGF-1R subcellular localisation for both subunits, but tested the response of only nuclear IGF-1R $\beta$-subunit to IGF-1R kinase inhibition [11]. Here, we again assessed the localisation of both subunits in serum-starved DU145 cells in the presence or absence of IGF-1. Immunofluorescence confirmed detection of IGF-1R in the nucleus with evidence that both IGF-1Ra-and IGF-1R $\beta$-subunits were IGF-1 responsive, showing a significant increase upon IGF-1 treatment (Fig. 1d), as previously shown [11]. In addition, we found evidence of significant IGF-1R membrane retention on treatment with IGF-1R tyrosine kinase inhibitor BMS-754807, with reduction in nuclear IGF-1 Ra-subunit and accumulation of IGF-1R on the plasma membrane (Fig. 1e) as previously seen with the IGF-1Rß-subunit [11] and also shown here (Fig. 1e). These data confirm our previous findings with respect to IGF-1R $\beta$-subunit translocation in DU145 cells [11], show that both IGF-1Ra- and $\beta$-subunits are detectable in the nucleus of $22 R v 1$ cells, and provide additional evidence that the a-subunit also undergoes IGF-induced nuclear translocation.

\subsection{Nuclear IGF-1R undergoes translocation from the cell surface}

We next wanted to check whether IGF-1R in the nucleus had originated from the cell surface. It is widely assumed that IGF$1 R$ undergoes ligand stimulation at the cell surface before translocation to the nucleus [11-15], but this has never been directly tested, although we have previously shown a reduction in nuclear IGF-1R $\beta$-subunit in DU145 cells in response to inhibition of clathrin-mediated endocytosis [11]. Given that the outer nuclear membrane is in continuity with the ER membrane, it has been proposed that some nuclear proteins could undergo nuclear import via direct lateral diffusion, the rapid process of lateral movement of proteins within a membrane [25].

To assess the route of IGF-1R trafficking, DU145 and 22Rv1 cells underwent biotinylation of cell surface proteins followed by ligand stimulation and subcellular fractionation. Biotinylated proteins were isolated from nuclear extract and probed by Western blot for presence of IGF-1 Ra- and $\beta$-subunits. The biotinylation labelling reagent is membrane impermeable and labels proteins via formation of an amide bond between the primary amine of lysine residues and the biotin label [26]. The IGF-1Ra-subunit is completely extracellular [1,3] and contains multiple primary amine residues that we determined would be labelled in the presence of the external biotinylation reagent. The IGF-1Rß-subunit is almost completely intracellular, although does contain a small extracellular domain (shown in Fig. 1a) that contains multiple 
lysine residues within the fibronectin type III (FnIII)-2b and -3 domains at Lys-782, -821, -838, -857 and -897 [ref. 1-4]. Therefore, both subunits were predicted to undergo biotinylation.

As expected, both subunits could be detected in nuclear, cytoplasmic and whole cell extract (WCE) protein input controls in both cell lines. Furthermore, both the $a$ - and $\beta$-subunits were detectable in nuclear extracts from cells that had undergone cell surface protein biotinylation. Neither subunit was detected in control extracts that had undergone incubation with PBS rather than biotinylation reagent (Fig. 2a, b). As a positive control, nuclear extracts were also shown to contain cell surface biotinylated epidermal growth factor receptor (EGFR), a protein well characterised to translocate from the cell surface to the nucleus [27-29]. In contrast, there was no detection of biotinylated lamin and, in 22Rv1, AR, which are both nuclear proteins that do not traffic from the cell surface. Given that only cell surface proteins had been exposed to biotinylation, the absence of both IGF-1R subunits in control samples and presence in nuclear extracts from surface biotin-labelled cells confirms that IGF-1R subunits detectable in the nucleus have translocated from the cell surface.

\subsection{IGF-1Ra- and $\beta$-subunits interact in the nucleus}

Following confirmation that nuclear IGF-1R had translocated from the cell surface, we next wanted to investigate whether the two subunits of the receptor retain their interaction in the nucleus. Following cellular fractionation of DU145 and 22Rv1 cells, $\alpha$ - or $\beta$-subunits were immunoprecipitated from nuclear extracts. As before, both subunits were detected in the nuclear, cytoplasmic and WCE protein input controls. Following immunoprecipitation from nuclear extract, the $\alpha$-subunit of IGF-1R could be detected in both DU145 and 22 Rv1 immunoprecipitates. The IGF$1 R \beta$-subunit could also clearly be detected following co-immunoprecipitation using a-subunit antibody. Reciprocal $\beta$-subunit immunoprecipitation also detected both IGF-1Ra- and $\beta$-subunits in DU145 and 22Rv1 nuclear extracts (Fig. 2c, d).

These experiments indicated interaction of IGF-1Ra- and $\beta$-subunits in the nucleus of prostate cancer cells. This finding could suggest that IGF-1R translocates as an $a_{2} \beta_{2}$ holoreceptor. The majority of RTKs that undergo nuclear import, including fibroblast growth factor and EGFR family members, translocate after juxta-membrane proteolytic release of cytoplasmic domain or nuclear import of full-length receptor monomers [27-29]. In contrast, IGF-1R and the closely related INSR are heterotetramers in which the $a_{2} \beta_{2}$ structure is maintained by disulphide bonds [3]. Nuclear INSR was reported originally by [30] and recently by Hancock et al. [31], the latter finding INSR interaction with RNAPollI, consistent with our data for IGF-1R [15], and showing by immunogold electron microscopy that a subset of nuclear INSRa- and $\beta$-chains were sufficiently close to each other for direct interaction [31]. Our finding of $a-\beta$ co-precipitation implies retention of disulphide-bonded subunits during trafficking from the endosomal compartment via the cytosol to the nucleus. The cytosol is a mildly reducing environment in which most thiols are in a reduced state but which can maintain disulphide-bonds in proteins transferred from other cellular compartments [32]. IGF-1R and INSR contain similar numbers of alpha and beta cysteines; mild INSR reduction is known to cleave a small number of disulphides, generating disulphide-linked $a \beta$ dimers that can still bind ligand, while more vigorous INSR reduction generates free alpha and beta chains [3]. Our detection of IGF-1R a- $\beta$ complexes in nuclear extract implies existence of similar IGF-1R a $\beta$ dimers or $\alpha_{2} \beta_{2}$ holoreceptors.

\subsection{IGF-1Ra is recruited to IGF-1R $\beta$ DNA binding sites}

Our previous ChIP-seq in DU145 cells revealed IGF-1R $\beta$ recruitment to regulatory regions of chromatin. At most of these sites, IGF-1R $\beta$ enrichment was coincident with recruitment of RNAPollI, and direct interaction between IGF-1R $\beta$ and RNAPolll was also detected. Both IGF-1R $\beta$ recruitment and RNAPolll interaction were shown to be IGF-dependent [15]. Here, having detected nuclear IGF-1 Ra- $\beta$ complexes (Fig. 2c, d), we investigated whether IGF-1R $\alpha$-subunit contributes to nuclear IGF-1R function. Therefore, we tested for IGF-1 Ra recruitment to two regions previously validated as sites of IGF-1R $\beta$ enrichment [15]. One is the promoter of the JUN oncogene which we confirmed to contribute to prostate cancer cell growth and motility [15], and the other an intergenic region of chromosome 17 (IntChr17) that is enriched for H3K27 acetylation but not RNAPolll enrichment (Fig. 3a), suggesting putative enhancer function [33].

As previously shown by our group [15], ChIP-qPCR in DU145 cells was able to detect significant recruitment of IGF$1 \mathrm{R} \beta$ to the JUN promoter (Fig. 3b), and here we also showed recruitment to IntChr17 (Fig. 3c), validating the ChIP-seq finding. At the JUN promoter region both subunits showed significant increase in recruitment versus IgG negative 
a

DU145

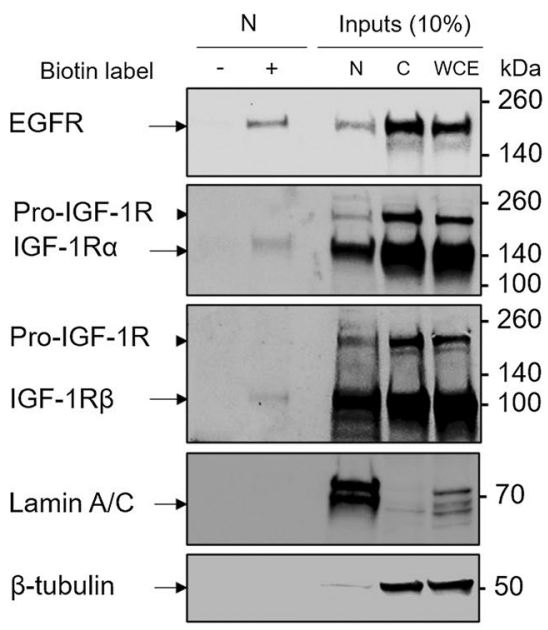

c

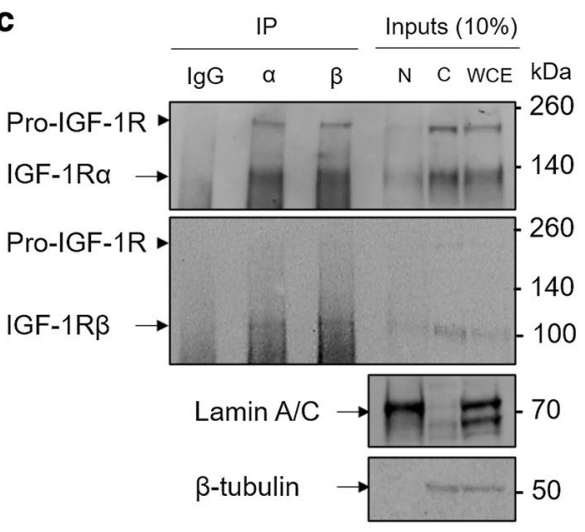

b

22Rv1

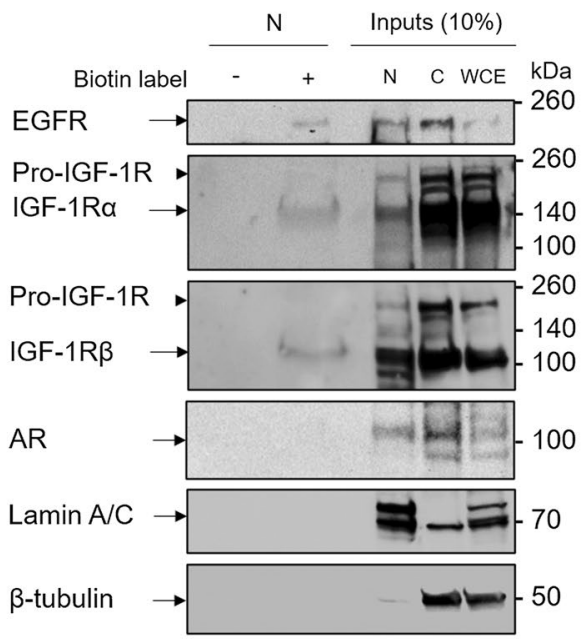

d

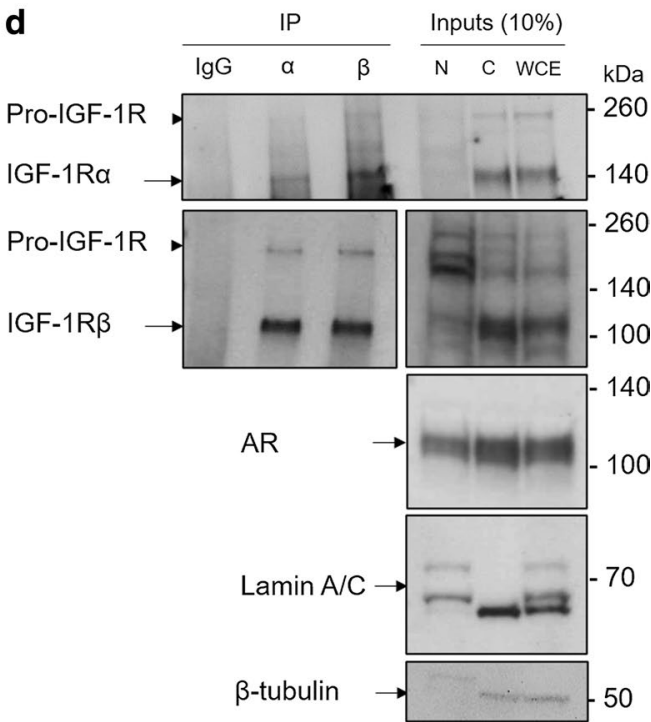

Fig. 2 Nuclear IGF-1Ra- and $\beta$-subunits originate from the cell surface and interact in the nucleus $\mathbf{a}, \mathbf{b}$ Western blots showing detection of cell surface biotinylated IGF-1R in the nucleus of DU145 (a) and 22Rv1 (b) cells. Detection of cell surface biotinylated EGFR in nuclear fractions acts as a positive control. Western blots shown are representative of 3 independent biological repeats. c, d Co-immunoprecipitation Western blot showing interaction of IGF-1Ra- and $\beta$-subunits in the nucleus of DU145 (c) and 22Rv1 (d) cells. Western blots shown are representative of 3 independent biological repeats

control, however a-subunit recruitment was found to be at a significantly lower level than that of the $\beta$-subunit (Fig. 3b). Although not shown here, to increase confidence in the specificity of this result, it would be helpful in future work to include ChIP-qPCR for an unrelated genomic region not previously found to be a site of IGF-1R $\beta$ recruitment. At the IntChr17 binding site, both subunits showed significant increase in recruitment at approximately equal levels (Fig. 3c). These results suggest that the IGF-1R a-subunit is also involved in recruitment of IGF-1R to chromatin. This mirrors the closely related INSR, which shares significant sequence homology, similar structure and overlapping functions with IGF-1R $[1,3,34,35]$, where recent work reported similar levels of recruitment of both $\alpha$ - and $\beta$ - INSR subunits to INSR chromatin binding sites validated by ChIP-qPCR [31].

It is worth noting that RNAPolll is not recruited to the IntChr17 region tested here, in contrast to the RNAPollI recruitment seen at the JUN promoter region [15] (Fig. 3a). As previously mentioned, our lab has shown direct interaction of RNAPollI and IGF-1R [15], therefore it is possible that this interaction is disrupting immunoprecipitation and 
a

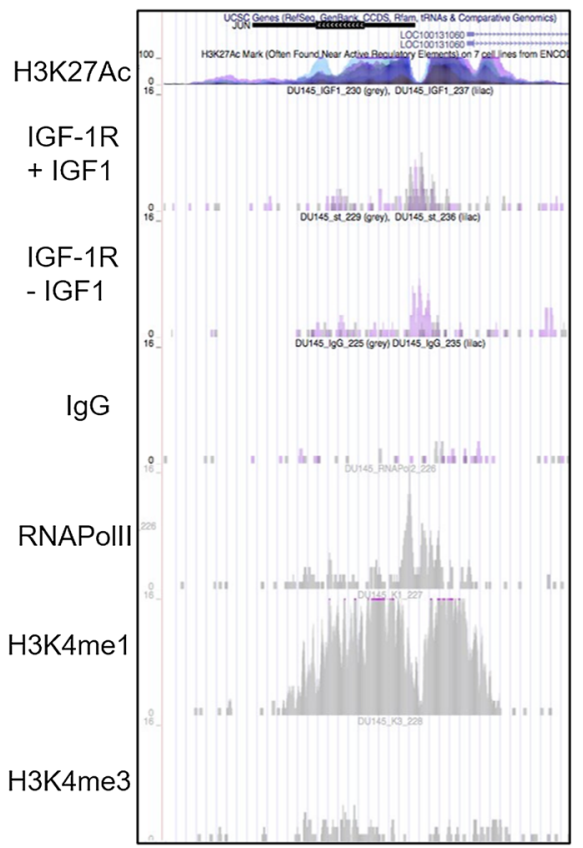

b ChIP-qPCR detection of recruitment to the JUN promoter region

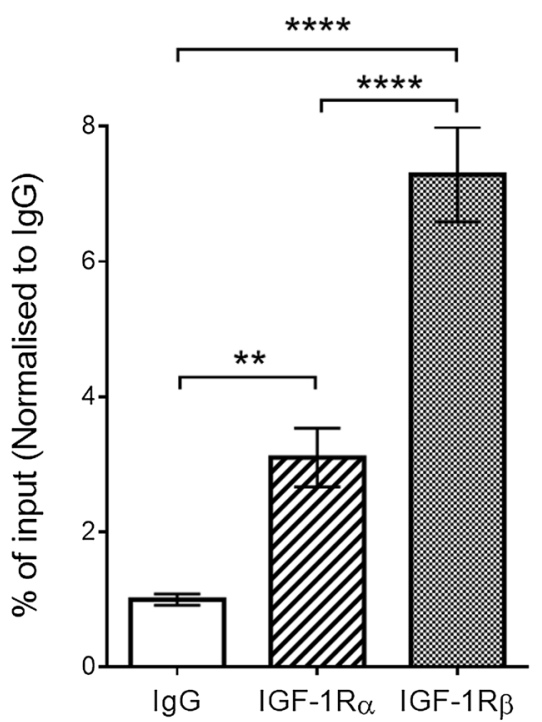

Chr17 Intergenic Region

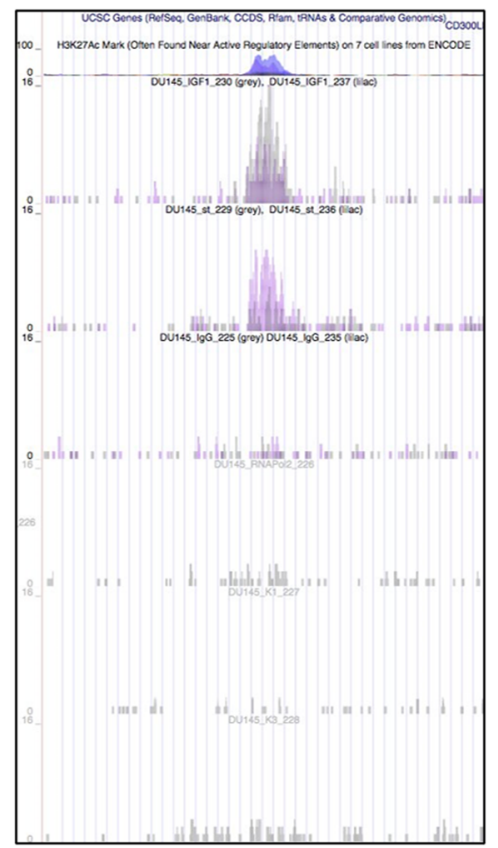

c ChIP-qPCR detection of recruitment to the Chr17 intergenic region

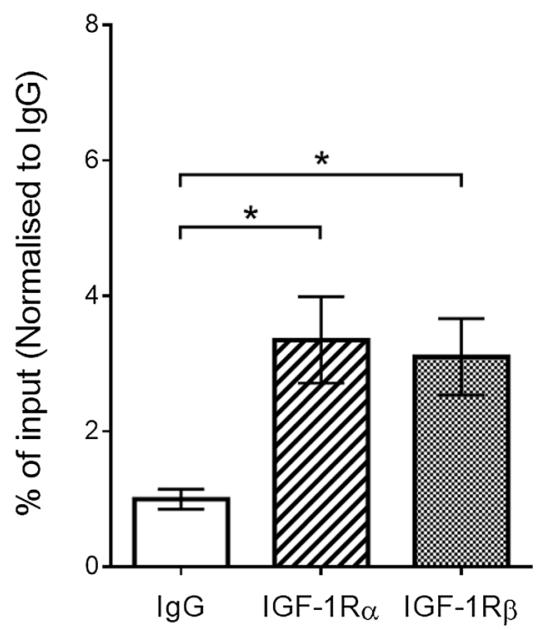

Fig. 3 Recruitment of IGF-1Ra is detectable at IGF-1R $\beta$ DNA binding sites. a Images from the UCSC genome browser of previously obtained ChIP-seq data showing IGF-1R binding peaks that denote recruitment of RNAPolll and IGF-1R $\beta$ to the JUN promoter and IntChr17 [ref 15]. b, c Quantification of recruitment of IGF-1Ra or IGF-1R $\beta$ vs IgG negative control to IGF-1R $\beta$ binding regions, detected by ChIP-qPCR. Results represent mean \pm SEM of triplicate independent experiments, each with 3 technical replicates. Recruitment of both IGF-1Ra and IGF-1R $\beta$ vs IgG was detected at the JUN promoter (b) and putative IntChr17 enhancer (c; $\mathrm{p}^{*}<0.05, \mathrm{p}^{* *}<0.005, \mathrm{p}^{* * * *}<0.0001$, Tukey's multiple comparisons test)

detection of the a-subunit at the JUN promoter region, resulting in differing levels of detected recruitment for the $a-$ and $\beta$-subunits at this site. These differences could also be due to technical factors such as different efficiency of antibodies for immunoprecipitation from chromatin, masking of DNA-interacting domain by antibody binding, and/ or interaction of the $a$-subunit indirectly via the $\beta$-subunit. 


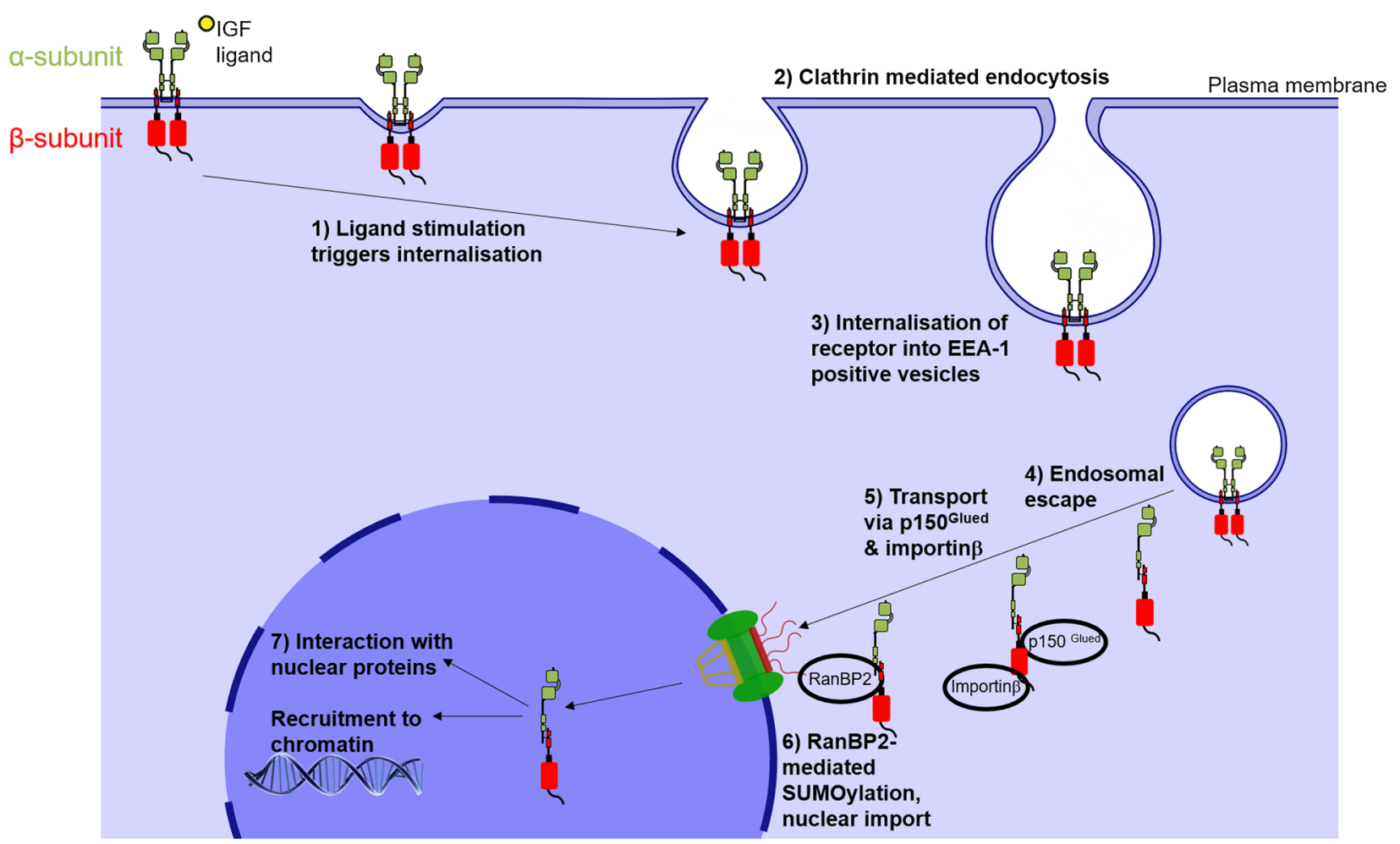

Fig. 4 Model of potential nuclear translocation mechanism of IGF-1R in its holoreceptor form 1-3. Ligand stimulation of IGF-1R triggers clathrin mediated endocytosis of the receptor into EEA-1 positive vesicles [11, 14]. 4. IGF-1R is presumed to undergo endosomal escape into the cytosol. Detection of nuclear IGF-1Ra- $\beta$ complexes in this manuscript suggests retention of disulphide bonded-subunits during transit through the cytosol. 5-6. Cytosolic IGF-1R interacts with p150 Glued and is transported to the nucleus via microtubule-dependent dynactin activity where it interacts with importin- $\beta$ followed by RanBP2. RanBP2 E3 SUMO ligase catalyses the tri-SUMOylation of 3 conserved lysine residues of the $\beta$-subunit $[12,14]$. 6. SUMOylation mediates IGF-1R translocation across the nuclear pore complex and into the nucleus. 7. Nuclear IGF-1R interacts with nuclear proteins including PCNA, nucleolar protein NOM1, transcriptional regulators including TCF/LEF1, Histone H3, RNAPollI, GATA-2 and with regulatory regions of chromatin to influence gene transcription and protein function [15-18, 36]

Taken together, we have shown that both IGF-1Ra- and $\beta$-subunits are detectable in the nucleus of $22 \mathrm{Rv} 1$ prostate cancer cells, building on previous results seen in DU145 cells [11]. Cell surface protein biotinylation experiments directly confirmed for the first time that IGF-1Rs detectable in the nucleus originate from the cell membrane, in line with a model in which the receptor undergoes translocation to the nucleus following ligand stimulation at the cell surface. We have also shown by co-immunoprecipitation that $\alpha$ - and $\beta$-subunits form nuclear complexes, suggesting that the receptor translocates as an $\alpha-\beta$ dimer or holoreceptor (Fig. 4). Finally, using ChIP-qPCR we detected a-subunit recruitment to a region of the JUN promoter and an intergenic region of Chromosome 17, previously identified as IGF-1R $\beta$ binding sites [15]. These findings suggest that IGF-1Ra-subunit is involved in IGF-1R chromatin binding and transcriptional activation of genes including $J U N$ that act as prostate cancer oncogenes, therefore contributing towards the more advanced tumour stage in prostate cancer and reduced overall survival in clear cell renal cancer associated with nuclear IGF-1R [11, 15].

While we have not directly assessed the prognostic value of nuclear IGF-1R signalling in this work, as mentioned above, we have previously found an association between nuclear IGF-1R and advanced tumour stage in patients undergoing radical prostatectomy. We also showed a correlation between nuclear IGF-1Rß-subunit and JUN in malignant prostate epithelium by immunohistochemistry [15]. However, as the patients from this cohort were generally a good prognostic group, it was not possible to assess association of nuclear IGF-1R with prostate cancer survival. To test the association of nuclear IGF-1R with prognosis and survival would require further work involving immunohistochemistry on large numbers of cases supported by good clinical data. Such datasets could also be used to test the correlation of JUN expression with IGF-1R activation via staining for phospho-IGF-1R. Future work is also needed to determine what further nuclear IGF-1R chromatin binding sites and protein interactions can now be detected via the a-subunit that may further elucidate the role of nuclear IGF-1R in cancer progression. 
Acknowledgements We would like to acknowledge the skills and expertise of the staff in the Microscopy Scientific Research Facility at the Department of Oncology, University of Oxford.

Authors' contributions All authors contributed to the study conception and design. Material preparation, data collection and analysis were performed by JVM. The first draft of the manuscript was written by JVM and all authors commented on previous versions of the manuscript. All authors read and approved the final manuscript.

Funding This study was supported by the Rosetrees Trust and John Black Foundation (M330-F1), Medical Research Council Industrial Strategy studentship (1718_MSD_1113081) to Jack V Mills, support to Valentine M Macaulay and Guillaume Rieunier from Cancer Research UK Early Detection Grant (C476/A27060) and support to Valentine M Macaulay from Prostate Cancer UK (RIA_ST2_024).

Data availability The data in this manuscript was generated from original work, and any and all raw data files can be made available on request. The IGF-1R ChIP-seq data set analysed to identify IGF-1R chromatin binding sites is available from the corresponding author upon reasonable request.

Code availability Not applicable.

\section{Declarations}

Competing interests The authors declare no competing interests.

Open Access This article is licensed under a Creative Commons Attribution 4.0 International License, which permits use, sharing, adaptation, distribution and reproduction in any medium or format, as long as you give appropriate credit to the original author(s) and the source, provide a link to the Creative Commons licence, and indicate if changes were made. The images or other third party material in this article are included in the article's Creative Commons licence, unless indicated otherwise in a credit line to the material. If material is not included in the article's Creative Commons licence and your intended use is not permitted by statutory regulation or exceeds the permitted use, you will need to obtain permission directly from the copyright holder. To view a copy of this licence, visit http://creativecommons.org/licenses/by/4.0/.

\section{References}

1. Ullrich A, et al. Insulin-like growth factor I receptor primary structure: comparison with insulin receptor suggests structural determinants that define functional specificity. EMBO J. 1986;5(10):2503-12.

2. Le Roith D, Werner H, Beitner-Johnson D, Roberts CT. Molecular and cellular aspects of the insulin-like growth factor I receptor. Endocr Rev. 1995;16(2):143-63.

3. Adams TE, Epa VC, Garrett TPJ, Ward CW. Structure and function of the type 1 insulin-like growth factor receptor. Cell Mol Life Sci. 2000;57:1050-93.

4. Li J, Choi E, Yu H, Bai X chen. Structural basis of the activation of type 1 insulin-like growth factor receptor. Nat Commun. 2019;10(1):1-11.

5. Baserga R, Peruzzi F, Reiss K. The IGF-1 receptor in cancer biology. Int J Cancer. 2003;107(6):873-7.

6. Resnicoff M, et al. The insulin-like growth factor I receptor protects tumor cells from apoptosis in vivo. Cancer Res. 1995;55(11):2463-9.

7. Osher E, Macaulay VM. Therapeutic targeting of the IGF axis. Cells. 2019;8(895):1-25.

8. Aleksic T, et al. Durable response of spinal chordoma to combined inhibition of IGF-1R and EGFR. Front Oncol. 2016;6(98):1-6.

9. Simpson A, Petnga W, Macaulay VM, Weyer-czernilofsky U, Bogenrieder T. Insulin-like growth factor (IGF) pathway targeting in cancer : role of the IGF axis and opportunities for future combination studies. Targ Oncol. 2017;12(5):571-97.

10. Baserga R. The decline and fall of the IGF-I receptor. J Cell Physiol. 2013;228(4):675-9.

11. Aleksic T, et al. Type 1 insulin-like growth factor receptor translocates to the nucleus of human tumor cells. Cancer Res. 2010;70(16):6412-20.

12. Sehat B, et al. SUMOylation mediates the nuclear translocation and signaling of the IGF-1 receptor. Sci Signal. 2010;3(108):1-12.

13. Sarfstein R, et al. Insulin-like growth factor-I receptor (IGF-IR) translocates to nucleus and autoregulates IGF-IR gene expression in breast cancer cells. J Biol Chem. 2012;287(4):2766-76.

14. Packham S, et al. Nuclear translocation of IGF-1R via p150 Glued and an importin- $\beta /$ RanBP2-dependent pathway in cancer cells. Oncogene. 2014;34(17):2227-38.

15. Aleksic T, et al. Nuclear IGF1R interacts with regulatory regions of chromatin to promote RNA polymerase II recruitment and gene expression associated with advanced tumor stage. Cancer Res. 2018;78(13):3497-509.

16. Solomon-Zemler R, Pozniak Y, Geiger T, Werner H. Identification of nucleolar protein NOM1 as a novel nuclear IGF1R-interacting protein. Mol Genet Metab. 2019;126(3):259-65.

17. Warsito D, Sjöström S, Andersson S, Larsson O, Sehat B. Nuclear IGF1R is a transcriptional co-activator of LEF1/TCF. EMBO Rep. 2012;13(3):244-50.

18. Waraky A, et al. Nuclear insulin-like growth factor 1 receptor phosphorylates proliferating cell nuclear antigen and rescues stalled replication forks after DNA damage. JBC. 2017;292(44):18227-39.

19. Asmane I, et al. Insulin-like growth factor type 1 receptor (IGF-1R) exclusive nuclear staining: a predictive biomarker for IGF-1R monoclonal antibody (Ab) therapy in sarcomas. Eur J Cancer. 2012;48(16):3027-35.

20. Hoa N, et al. Nuclear targeting of IGF-1 receptor in orbital fibroblasts from Graves' disease: apparent role of ADAM17. PLoS ONE. 2012;7(4):1-9.

21. Massie $\mathrm{CE}$, et al. The androgen receptor fuels prostate cancer by regulating central metabolism and biosynthesis. EMBO J. 2011;30(13):2719-33.

22. Heinlein CA, Chang C. Androgen receptor in prostate cancer. Endocr Rev. 2004;25(2):276-308. 
23. Turney BW, Turner GDH, Brewster SF, Macaulay VM. Serial analysis of resected prostate cancer suggests up-regulation of type 1 IGF receptor with disease progression. BJU Int. 2011;107(9):1488-99.

24. Krueckl SL, et al. Increased insulin-like growth factor I receptor expression and signaling are components of androgen-independent progression in a lineage-derived prostate cancer progression model. Cancer Res. 2004;64(23):8620-9.

25. Ungricht $R$, Klann $M$, Horvath $P$, Kutay U. Diffusion and retention are major determinants of protein targeting to the inner nuclear membrane. J Cell Biol. 2015;209(5):687-704.

26. Pierce ${ }^{T M}$ Cell Surface Protein Biotinylation and Isolation Kit User Guide. ThermoScientific. 2020.

27. Lin S, et al. Nuclear localization of EGF receptor and its potential new role as a transcription factor. Nat Cell Biol. 2001;3(9):802-8.

28. Bryant DM, Stow JL. Nuclear translocation of cell-surface receptors: Lessons from fibroblast growth factor. Traffic. 2005;6(10):947-53.

29. Wang SC, Hung MC. Nuclear translocation of the epidermal growth factor receptor family membrane tyrosine kinase receptors. Clin Cancer Res. 2009;15(21):6484-9.

30. Nelson JD, LeBoeuf RC, Bomsztyk K. Direct recruitment of insulin receptor and ERK signaling cascade to insulin-inducible gene loci. Diabetes. 2011;60(1):127-37.

31. Hancock ML, et al. Insulin receptor associates with promoters genome-wide and regulates gene expression. Cell. 2019;177(3):722-36.

32. Sevier CS, Kaiser CA. Formation and transfer of disulphide bonds in living cells. Nat Rev Mol Cell Biol. 2002;3(11):836-47.

33. Calo E, Wysocka J. Modification of enhancer chromatin: what, how, and why? Mol Cell. 2013;49(5):825-37.

34. Brzozowski AM, et al. Structural origins of the functional divergence of human insulin-like growth factor-I and insulin. Biochemistry. 2002;41(30):9389-97.

35. De Meyts P, Whittaker J. Structural biology of insulin and IGF1 receptors: Implications for drug design. Nat Rev Drug Discov. 2002;1(10):769-83.

36. Warsito D, Lin Y, Gnirck AC, Sehat B, Larsson O. Nuclearly translocated insulin-like growth factor 1 receptor phosphorylates histone $H 3$ at tyrosine 41 and induces SNAI2 expression via Brg1 chromatin remodeling protein. Oncotarget. 2016;7(27):42288-302.

Publisher's Note Springer Nature remains neutral with regard to jurisdictional claims in published maps and institutional affiliations. 